\title{
Bronchoscopic treatment options for chronic obstructive pulmonary disease
}

\author{
Şehnaz OLGUN YILDIZELI
}

\begin{abstract}
Chronic obstructive pulmonary disease (COPD) is a major cause of high mortality and morbidity worldwide. Symptoms of the disease are usually related with the results of pulmonary dynamic hyperinflation. Treatment options are classified as invasivenoninvasive and mostly tend to be palliative. Lung volume reduction techniques are invasive part of the treatment and can be performed by surgical or bronchoscophic methods. Because of the low complication rates bronchoscophic methods became more popular in recent years. Patients presenting with severe air trapping and thoracic hyperinflation have the greatest potential to derive benefit from volume reduction procedures. Assessment of patients should ideally include cardiological evaluation, high resolution CT scan, ventilation and perfusion scintigraphy, full pulmonary function tests, and cardiopulmonary exercise testing.

There are three main groups of bronchoscophic volume reduction, direct effect on lung parenchyma, airway bypass and blocking the airways. Indications and contraindications are different for each modality and device. Patients who have deterioration despite habitual changes and medical therapies should be evaluated for volume reduction procedures.
\end{abstract}

Keywords: COPD, Emphysema, Hyperinflation, Lung volume reduction, Bronchoscopic

Sehnaz Olgun Yildizeli

Department of Pulmonary and Critical Care Medicine, School of Medicine, Marmara University, Istanbul, Turkey

\section{Introduction}

Chronic obstructive pulmonary disease (COPD) causes high mortality and morbidity worldwide. It is estimated that 65 million people suffer from COPD and it is expected that disease will be third most common cause of death in 2020 [1]. It will also be the fifth cause of Disability Adjusted Life Years (DALY) lost by the year 2020 [2].

Symptoms of the disease such as shortness of the breath and reduced exercise capacity are usually results of irreversible bronchial constriction and dynamic pulmonary hyperinflation [3]. Emphysema form of disease is characterized by abnormal and permanent enlargement of the airspaces and usually results in reduced transpulmonary recoil pressures and chest wall mechanics. These changes lead to hypercapnia and hypoxia that often require oxygen support [4]. Risk factors for COPD are known as smoking, biomass exposure to organic and inorganic chemical agents, poor socioeconomic status, bronchial asthma, bronchial hypersensitivity, chronic bronchitis, early childhood infections, and tuberculosis [5].

\section{Treatment options for COPD}

Treatment options for COPD (invasive and noninvasive) tend to be palliative rather than curative. Noninvasive treatment options are cessation of tobacco, prophylactic vaccination against to pneumococci and influenza, respiratory therapy and medical therapy that contains anticholinergics, B2 sympathomimetics, inhaled glucocorticosteroids, phosphodiesterase inhibitors mucolytics, antioxidants and nutritive support to protect from muscle atrophy. Patients with chronic hypoxemic respiratory insufficiency may also utilize long term oxygen therapy and noninvasive mechanical ventilation. Management of the medical therapy depends on evidence based 'Global Initiative for Management of Obstructive Lung Diseases' (GOLD) that classify severity of 
the diseases according to post-bronchodilator $\mathrm{FEV}_{1}$ values [5]. According to GOLD guideline cessation of tobacco is single intervention that modifies lung function and increases survival [5]. Despite these therapies most of the patients with moderate to severe illness have minimal or no control on disease or deteriorate rapidly that make them a candidate for lung transplantation or for other invasive procedures [6].

Surgical management of emphysema was first described in 1954 as removal of the hyperinflated and physiologically nonfunctional parts of the lung [7]. Because of postoperative persistent air leaks and high mortality rates operation did not become popular until end of 1990s. In 2003, 'The National Emphysema Treatment Trial' reported the post operative high mortality ( $7.9 \%$ within 90 days) in surgery group, however they showed improvement in exercise capacity and quality of life with predominantly upper lobe emphysema [8]. But still because of high mortality, restriction of patient selection criteria, cost and effectiveness, medical communities did not approve surgical lung volume reduction (LVR). Because of these disadvantages brochoscophic lung volume reduction (BVR) developed in recent years.

The principle of BVR is the same as LVR surgery with low complication rates [9]. According to their mechanism there are three main groups of BVR techniques: methods that have direct effect on lung parenchyma (polymeric lung volume reduction, coils and thermal vapor ablation), methods that facilitate the expiration of trapped air from the emphysematous lung(airway bypass) and methods employing devices that block the airways (spigots and unidirectional valves) [10]. Patients with diagnosis of hyperinflation in chest X Ray or pulmonary computed tomography (CT), forced expiratory volume in one seconds $\left(\mathrm{FEV}_{1}\right)$ values less than $500 \mathrm{~mL}$, arterial partial oxygen pressure $\left(\mathrm{PaO}_{2}\right)$ less than $50 \mathrm{mmHg}$ and arterial carbon dioxide partial pressure $\left(\mathrm{PaCO}_{2}\right)$ more than $50 \mathrm{mmHg}$ are candidates for BLVR procedure. Patients should quit smoking and must enroll in a pulmonary physiotherapy program [11]. Comparing with LVR, this procedure can be performed under sedation, general anesthesia is not obligatory.

Recently, completed multicenter studies show that not all classes and phenotypes of emphysema benefit from BVR, and that each technique appears to provide a greater benefit to specific subgroup of patients, because of the high cost of the procedures selections must be done carefully. The initial screening for suitable candidates should be performed at the pulmonologist level on patients with stable disease and no recent exacerbations.
The evaluation should include a through history (level of dyspnea, functional impairment, past thoracic surgery, comorbidities and smoking status) and physical examination. Thorax CT especially HRCT is essential to see the heterogeneity, fissure integrity, degree of destruction and ruling out underlying diseases such as lung cancer. Other tests are pulmonary function tests (full parameters including RV, TLC, DLCO), 6-minute walking test (6MWT), arterial blood gases, echocardiography should be completed before finalizing BVR decision. BVR should not be offered to active smokers, patients with pulmonary hypertension, unstable cardiac pathology, active respiratory infections or a very poor exercise tolerance, patients with no clear evidence of hyperinflation or patients who are on any type of antiplatelet or anticoagulant therapy which cannot be stopped for 7 days prior to the procedure [12].

\section{Indications}

- 40-75 years

- Heterogeneous emphysema and no collateral ventilation (valves)

- Dyspnea (Despite maximal medical therapy and pulmonary rehabilitation)

- $\mathrm{FEV}_{1} 15-45 \%$

- Hyperinflation with TLC $>100 \%$ and RV $>150$ $175 \%$

- $\mathrm{PaCO}_{2}<50 \mathrm{mmHg}$

- $\mathrm{PaO}_{2}>45 \mathrm{mmHg}$ (while breathing ambient air)

- $6 \mathrm{MWD}>140 \mathrm{~m}$

\section{Contraindications}

- Homogeneous emphysema (valves)

- Collateral ventilation /non-intact fissures

- $\quad>75 \%$ parenchymal destruction on HRCT (coils)

- Current smoking (at least quit 6 months)

- $\mathrm{DLCO}<20 \%$

- Giant bullae (>1/3 of hemithorax)

- Alfa-1 Antitrypsin deficiency

- Previous thoracotomy, pleurodesis

- Chest wall deformity

- Excessive sputum 
- Severe pulmonary Hypertension $(>50 \mathrm{mmHg})$

- Active infection

- Unstable cardiac conditions

- Significant pleural or interstitial changes on HRCT

- Any type of platelet or anticoagulant therapy which cannot be stopped for 7 days prior to the procedure

\section{Modalities and Devices}

\section{Unidirectional Endobronchial and Intrabronchial Valves}

Unidirectional endobronchial valves are certainly the most widely studied devices for the bronchoscopic treatment of emphysema also have the largest series of treated patients.

There are two different types of valves available on the market Zephyr valves (Pulmonx, Inc., CA, USA) and IBV (Spiration Inc., Washington, USA). These devices are self-expanding and delivered with a working channel of a flexible bronchoscope.

Zephyr valves are made of nitinol (nickel titanium) mesh covered by silicone and contains double silicone membrane inside that opens during expiration and closes during inspiration [10]. Zephyr valves are available in two sizes, one for segmental (4.0-9.0 mm) and one for lobar bronchi (5.5-8.5 $\mathrm{mm}$ ), anchorage of the valve to the airway is facilitated by the irregular surface and self-expanding strength of the mesh.

Intrabronchial valves are umbrella-shaped devices made of a nitinol mesh covered by a polyurethane membrane [10]. The valve is secured to the airway wall by hook-like anchors. It has 4 different sizes (5,6,7 and $9 \mathrm{~mm}$ ), airways are sized with a balloon prior to deployment of valves with the aid of a flexible bronchoscope.

Endobronchial (EBV) and intrabronchial valves are the most extensively studied devices for BVR. Initial studies showed improvement on lung function and exercise tolerance and also safety of procedure. Endobronchial Valve for Emphysema palliation Trial (VENT) was a large randomized multicenter study that totally 492 patients were enrolled. The patients were randomized to valve treatment arm (unilateral volume reduction) and standard medical care [13]. Six months later improvement on $\mathrm{FEV}_{1}$ and 6MWT were detected in EBV group. High heterogeneity emphysema and intact fissures were related with high success rates but complications after EBV procedure were significantly higher than medical treatment arm. Recently Ninane et al. published a European multicenter study to asses safety and effectiveness of bronchial valves [14]. Results showed that procedure and devices were well tolerated but treatment without complete lobar occlusion in both upper lobes was ineffective in most of the patients. After this study Eberhardt et al. confirmed that unilateral intrabronchial valve placement with complete occlusion is superior to bilateral partial occlusion [15]. Finally STELVIO study showed that the lack of collateral ventilation is essential for the success of BVR with valves [16].

\section{Coils}

Nitinol spring-like coils in a straightened configuration are passed through the working channel of a flexible bronchoscope into subsegmental airways (PneumRx). When deployed, coil up and tether the lung. They have been designed for patients with either homogeneous or heterogeneous emphysema. It is available in 3 lengths (100, $125,150 \mathrm{~mm})$ and implanted via a flexible bronchoscope under general anaesthesia or conscious sedation with a delivery system under fluoroscopic guidance. The airway in the selected segment is identified with a low -stiffness guidewire (under fluoroscopy), after which a catheter is passed over the guidewire and length of the airway is measured. After removing guidewire a straightened coil is introduced into the distal end of catheter with a grasper, after which the catheter is removed while the proximal end of the coil is initially advanced and then released, assuming its performed shape ${ }^{12}$. The coils are purposed to work independently of collateral ventilation. The major adverse events are mild hemoptysis, chest pain, COPD exacerbation and usually occur in the first month of the procedure [17].

A pilot study by Herth et al. found BVR with coils to be safe and feasible [17]. In a multicenter study by Shah et al., 47 patients with homogeneous or heterogeneous severe emphysema were randomized for coil treatment or best medical care and primary endpoint was the difference in response on the St. George's Respiratory Questionnaire (SGRQ) between the treatment and usual care group at 90 days later [18]. The SGQR response at 90 days after final treatment was greater than in the usual care group and $>$ $60 \%$ of the patients in BVR group experienced improvement in $\mathrm{FEV}_{1}$ and no significant adverse event was reported. It is also confirmed that BVR using coils are safe and with no late pneumothoraces, coil migration or unexpected adverse events by Hartman et al. [19]. According to this study, 3 
years after treatment $50 \%$ of the patients maintained an improvement 6MWT, SGRQ and dyspnea scores.

Current evidence would therefore suggest that both appropriate candidates with heterogeneous and those with homogeneous emphysema could experience clinically significant benefits from BVR using coils, irrespective of collateral ventilation or complete lobar collapse.

\section{Bronchial Plugs/Spigots}

Watanable Spigots (Novatech) is a treatment option for persistent pneumothorax, pulmonary fistula and continuous air leakage [12]. There are three different sizes of plugs and made from silicon in conical shape. Reports about persistent air leakage are successful but evidence for BVR are limited. Toma et al., reported improvement in quality of life and exercise capacity after treatment in a small group, they also reported pneumonia as a complication due to failure of cleaning secretions [12].

\section{Sealants}

These products (hydrogel) are developed to obtain atelectasis and fibrosis of the lung parenchyma [10]. Biological substances have now replaced by synthetic polymeric foam (Aeris therapeutics), the foam flows into the peripheral airways and acts as a glue that seals the target regions with consequent atelectasis [10]. It blocks inter-alveolar as well as bronchiolar-alveolar collaterals, thus it will negate the effect of collateral ventilation. This procedure is irreversible and common side effects are COPD exacerbation, self-limiting systemic inflammatory response with infiltrates on chest X-Ray. Recently a multicenter study to evaluate effectiveness of polymeric foam was terminated early because of severe adverse events (up to $44 \%$ of patients) resulting with death despite confirmed significant improvements in lung function, dyspnea and lung function tests [20].

\section{Airway Bypass Stents}

This technique entails creation of an airway bypass passage between hyper-inflated lung tissue and bronchus such that the over inflated lobe will empty via bypass, with decrease in residual volume RV and air trapping [21]. First step of the procedure is to detect an area free of blood vessels by using a Doppler ultrasound, second step is fenestration of the airway and dilatation by a needle and final step is deploying a paclitaxel-coated stent to keep the passage open [22]. Current evidence suggest that although some short-term benefit may be achieved in patients with severe hyperinflation, the trends is for pulmonary functions to return to baseline overtime [21].

\section{Thermal Vapor Ablation}

Bronchoscopic thermal vapor ablation (Inter Vapor Uptake Medical) uses high-temperature vapor delivered into the target lung segments through a catheter with precise amount of energy thereby inducing thermal damage and inflammatory reaction resulting in permanent airway fibrosis [10]. This technique is also irreversible and not limited by collateral ventilation. Data is limited for vapor ablation technique but recently two studies published improvement in clinical outcomes [12]. Major frequent adverse events reported as COPD exacerbation and pneumonia.

Current evidence suggest that in well-defined sub-groups of patients with severe emphysema BVR may be of benefit. A well-structured approach to BVR, including initial screening and subsequent referral to specialized center is important to ensure against inappropriate use of devices, which may be both wasteful and harmful.

Patients with a high degree of hyperinflation and relatively preserved lung parenchyma are more likely to benefit from BVR using bilateral coils (irrespective of the collateral circulation and heterogeneity of the disease) whereas patients with heterogeneous disease, no collateral circulation and low baseline perfusion may benefit from unilateral BVR using valves with the goal of achieving lobar collapse. The exact utility of BVR with valves and/ or coils in homogeneous disease or the use of other devices, particularly sealants is still being investigated [12].

\section{References}

1. Murray CJ, Lopez AD. Measuring the global burden of disease. N Engl J Med 2013;369:448-57.

2. Lopez AD, Murray CC. The global burden of disease, 19902020. Nat Med 1998;4:1241-3.

3. Celli BR, Cote CG, Marin JM, et al. The body-mass index, airflow obstruction, dyspnea, and exercise capacity index in chronic obstructive pulmonary disease. N Engl J Med 2004;350:1005-12.

4. Lopez AD, Shibuya K, Rao C, et al. Chronic obstructive pulmonary disease: current burden and future projections. Eur Respir J 2006;27:397-412. 
5. Guidelines GlfCOLDG. Factors that influence disease development and progression. 2014:4-6.

6. Meena M, Dixit R, Singh M, Samaria JK, Kumar S. Surgical and bronchoscopic lung volume reduction in chronic obstructive pulmonary disease. Pulm Med 2014;2014:757016.

7. Brantigan OC. The surgical treatment of pulmonary emphysema. W V Med J 1954;50:283-5.

8. Fishman A, Martinez F, Naunheim K, et al. A randomized trial comparing lung-volume-reduction surgery with medical therapy for severe emphysema. N Engl J Med 2003;348:2059-73.

9. Sabanathan S, Richardson J, Pieri-Davies S. Bronchoscopic lung volume reduction. J Cardiovasc Surg 2003;44:101-8.

10. Gasparini S, Zuccatosta L, Bonifazi M, Bolliger CT. Bronchoscopic treatment of emphysema: state of the art. Respiration; International Review of Thoracic Diseases 2012;84:250-63.

11. Cohen E. Bronchoscopic treatment of end-stage chronic obstructive pulmonary disease. Curr Opin Anaesthesiol 2014;27:36-43.

12. Koegelenberg CF, Slebos DJ, Shah PL, et al. Time for the Global Rollout of Endoscopic Lung Volume Reduction. Respiration; International Review of Thoracic Diseases 2015;90:430-40.

13. Strange C, Herth FJ, Kovitz KL, et al. Design of the Endobronchial Valve for Emphysema Palliation Trial (VENT): a non-surgical method of lung volume reduction. BMC Pulm Med 2007;7:10.

14. Ninane V, Geltner C, Bezzi M, et al. Multicentre European study for the treatment of advanced emphysema with bronchial valves. Eur Respir J 2012;39:1319-25.
15. Eberhardt R, Gompelmann D, Schuhmann M, Heussel CP, Herth FJ. Complete unilateral vs partial bilateral endoscopic lung volume reduction in patients with bilateral lung emphysema. Chest 2012;142:900-8.

16. Klooster K, Ten Hacken NH, Hartman JE, Kerstjens HA, van Rikxoort EM, Slebos DJ. Endobronchial Valves for Emphysema without Interlobar Collateral Ventilation. N Engl J Med 2015;373:2325-35.

17. Herth FJ, Eberhard R, Gompelmann D, Slebos DJ, Ernst A. Bronchoscopic lung volume reduction with a dedicated coil: a clinical pilot study. Ther Adv Respir Dis 2010;4:225-31.

18. Shah PL, Zoumot Z, Singh S, et al. Endobronchial coils for the treatment of severe emphysema with hyperinflation (RESET): a randomised controlled trial. The Lancet Respir Med 2013;1:233-40.

19. Hartman JE, Klooster K, Ten Hacken NH, Slebos DJ. Treatment of emphysema using bronchoscopic lung volume reduction coil technology: an update on efficacy and safety. Ther Adv Respir Dis 2015;9:251-9.

20. Come CE, Kramer MR, Dransfield MT, et al. A randomised trial of lung sealant versus medical therapy for advanced emphysema. Eur Respir J 2015;46:651-62.

21. Choong CK, Cardoso PF, Sybrecht GW, Cooper JD. Airway bypass treatment of severe homogeneous emphysema: taking advantage of collateral ventilation. Thorac Surg Clin 2009;19:239-45.

22. Taneja A. Bronchoscopic interventions in the management of chronic obstructive pulmonary disease. Curr Opin Pulm Med 2013;19:145-51. 